\title{
Failure and detachment path of impulsively loaded plates
}

\author{
Dario Schiano Moriello*, Federico Bosi, Ryo Torii, PJ Tan \\ Department of Mechanical Engineering, University College London, Torrington Place, \\ London WC1E 7JE
}

\begin{abstract}
The deformation and detachment path of simply-supported and fully clamped mild steel quadrangular plates subjected to impulsive blast loads are investigated numerically. A comprehensive failure model that incorporates two competing mechanisms of damage due to ductile and shear failure is employed to simulate the progression of tearing within the plate. The stress triaxiality dependent Modified Mohr-Coulomb (MMC) fracture criterion is implemented in a finite element model, and experimental tensile and shear tests were carried out to calibrate the material parameters. The direction of crack propagation along the clamped plate support and the residual length are predicted for a wide range of impulse intensity, reproducing the failure modes observed experimentally. Furthermore, the developed model highlights the different failure mechanisms that occur in simply-supported plates, similar to those of panels subjected to localised blast loadings. Parametric studies are performed to establish dimensionless failure maps and investigate the influence of plate topology and boundary conditions on the dynamic response, for both square and rectangular geometries, thus offering an effective support for the design of impulsively loaded plates for advanced engineering applications.
\end{abstract}

Keywords: metal plates, failure modes, crack propagation, tear length, blast loading

\footnotetext{
* Corresponding author

Email address: dario.schiano.15@ucl.ac.uk (Dario Schiano Moriello)
}

Preprint submitted to Thin-Walled Structures

July 13, 2020 


\section{Introduction}

The prediction of the inelastic response of ductile structures under explosive loadings is a paramount concern in the fields of industrial, military or civil engineering. Metal plates, usually made of steel or aluminium alloys, constitute 5 a common structural component, and the analysis of their modes of deformation and failure is a topic of ongoing study [1, 2, 3. Teeling-Smith et al. 4, and Nurick et al. [5] have identified the three most common responses of clamped plates to uniform blasts. Ordered by loading intensity, they are summarised as: failure mode I, large inelastic deformation; mode II, inelastic deformation with material rupture due to tensile loading; mode III, rupture due to transverse shear with small deformation. Failure modes II and III are characterised by the detachment of part of the structure from its supports, causing the creation of projectiles, with obvious implications on structural safety. An additional failure mode is occasionally observed at the mode I-II transition, in which partial

15 tearing occurs at the boundary edge without detachment, indicated as mode II* [5. Numerous experimental studies have been conducted to investigate the plate behaviour for various geometries (i.e. square [5, 6, rectangular [3, 7] or circular [4, 8, plates), boundary conditions [3, 9] or stiffener configuration [10, 11]. In particular, an alternative sequence of failure modes has been identified when a plate is subjected to locally distributed blast loadings. Under these conditions, mode II $^{*}$ is replaced by failure modes with partial or complete tearing in the central area, labelled as mode $\mathrm{II}^{*} \mathrm{c}$ and mode IIc, respectively [2, 3, 8, 11].

Analytical models were developed to predict the amount of permanent deformation at the plate centre in the absence of failure [12. With the hypothesis of rigid, perfectly plastic material, the maximum transverse deflection in mode I can be determined as a function of the blast velocity, surface area and thickness.

Extensive experimental programs, generally time consuming and expensive, are necessary to assess in detail the deformation mode of plates when material failure occurs. Commercial finite element (FE) packages provide a valid, 
cost-effective alternative to experimental programs. Rudrapatna et al. [10, 13] employed FE models to predict the post-failure response of square plates deforming in mode II and III, taking into account material strain-rate sensitivity and material and geometrical non-linearities. An interactive failure criterion between membrane strains and transverse shear stress was proposed to predict the various failure modes, and the progression of fracture along the boundaries was modelled with a node-release algorithm. Similarly, Yuan et al. [14] used an FE model with simplified damage criteria to predict the behaviour of rectangular plates, and employed fictitious boundary conditions to guarantee stress convergence along the support. More recently, numerical studies that also included the

40 effect of thermal softening were used to study the transient deformation prior to failure [2] and the effect of the explosive stand-off distance [15]. However, most FE analyses employ oversimplified models of damage mechanisms, calibrated only on data from tensile tests, failing to represent accurately the influence of the loading conditions on material ductility.

Recent studies have been trying to solve fracture mechanics problems with alternative numerical approaches. For instance, Imachi et al. [16] introduced a peridynamic approach based on dynamic stress intensity factors for the analysis of crack propagation in brittle materials. Nguyen et al. [17] employed a nonlocal strain gradient damage model in FEM, coupled with elastic hardening ${ }_{50}$ and progressive softening material laws, for the fracture analysis of quasi-brittle materials. Wu et al. 18 proposed a mesh-free particle Galerkin method to simulate metal grinding processes. The method implements a bond-based failure criterion, together with a strain hardening law, where neighbouring particles are disconnected when their strain reaches a critical value.

In the present work, FE simulations are carried out in the commercial FE package Abaqus/Explicit (Dassault Systèmes [19]), implementing more comprehensive damage models [20, 21, with the scope of examining the evolution of the detachment path and the influence of the loading intensity and boundary conditions on the failure modes of metal plates loaded impulsively. The model 60 parameters are obtained from experimental data from tensile and shear tests, to 
characterise the dependence of material ductility on the loading conditions. The damage variables are opportunely calibrated to ensure the mesh insensitivity of the failure mechanism.

The study explores the failure modes of both clamped and simply supported plates, identifying a previously unobserved 'ripping' mode for rectangular plates constrained by the latter boundary conditions. In addition, parametric studies are performed to develop failure maps, detailing the effects of plate geometry on the dynamic response, specifically, of the plate thickness and surface area for square plates, and of the aspect ratio for rectangular plates.

The paper is organised as follows: the material model and the experimental characterisation are detailed in Sec. 2. Sec. 3 reports the FE model and its validation against experimental data available in the literature. A new criterion for identifying the transition between failure modes II-III is also proposed. Finally, the numerical results are discussed in Sec. 4, where the predictions of the parametric studies are presented in the form of failure maps for plate thickness, aspect ratio and boundary conditions.

\section{Material characterisation and damage models}

The present work employs a constitutive model for metal ductility, developed in the framework of $J_{2}$ plasticity, combined with two concurring continuum damage mechanics models for the representation of ductile failure caused by void nucleation, growth and coalescence, and shear failure induced by shear band localisation. Ductile damage initiation is represented by a function that correlates the equivalent plastic strain at necking $\bar{\varepsilon}_{0}$ with stress triaxiality $\eta$ 20, 21]:

$$
\bar{\varepsilon}_{0}=\bar{\varepsilon}_{0}(\eta)
$$

Stress triaxiality is a variable used to identify the three-dimensional loading state within the body, defined as the ratio of hydrostatic pressure $p$ to the von Mises stress $\bar{\sigma}$ :

$$
\eta=-\frac{p}{\bar{\sigma}},
$$


where $\eta=0$ identifies pure shear, $\eta=1 / 3$ represents uniaxial tension, and $\eta=2 / 3$ denotes equibiaxial tension.

The Modified Mohr-Coulomb (MMC) damage initiation criterion [20, 22] was chosen for its effectiveness in the range of low triaxiality, necessary for an accurate prediction of failure mode III, which is dominated by shear. Under the assumption of plane stress, the MMC criterion can be expressed as [22]

$\bar{\varepsilon}_{0}=\left\{\frac{A}{c_{2}}\left[c_{3}+\frac{\sqrt{3}}{2-\sqrt{3}}\left(1-c_{3}\right)\left(\frac{1}{\cos f_{\eta}}-1\right)\right]\left[\cos f_{\eta} \sqrt{\frac{1+c_{1}^{2}}{3}}+c_{1}\left(\eta+\frac{\sin f_{\eta}}{3}\right)\right]\right\}^{-\frac{1}{m}}$,

where

$$
f_{\eta}=\frac{1}{3} \arcsin \left[-\frac{27}{2} \eta\left(\eta^{2}-\frac{1}{3}\right)\right] .
$$

The definition of the fracture locus of Eq. (3) requires the calibration of five material parameters, $A, m, c_{1}, c_{2}, c_{3}$, starting from experimental data of $\bar{\varepsilon}_{0}$ for a wide range of loading conditions. The original calibration procedure proposed by Luo and Wierzbicki [23] is based on material tests in tensile, shear, compression and in combined tensile and shear loading conditions. An alternative experimental approach was proposed by Brünig et al. 24] and Driemeier et al. [25, solely based on the tensile testing of notched prismatic coupons. Different loading conditions, corresponding to distinct values of $\eta$, can be obtained at the specimen mid-length depending on the notch radius, as described in Appendix A.

Following the latter procedure, experiments were carried out in an electromechanical testing machine (Instron, model 5985) at a constant cross-head speed of $0.05 \mathrm{~mm} / \mathrm{s}$. Five tests were performed for each geometry. The experimental force-elongation $(F-\Delta)$ curves for each notch radius are presented in Fig. 1a The label $\mathrm{R} x$ indicates notched specimens, where $x$ specifies the notch radius in $\mathrm{mm}$ ( $\mathrm{R} 0$ indicates the uniaxial tensile test on unnotched samples), while the label S represents the force-elongation curve in pure shear.

The experiments were then reproduced in Abaqus/Standard [19] to numerically measure the values of stress triaxiality $\eta$ at the point of necking associated 


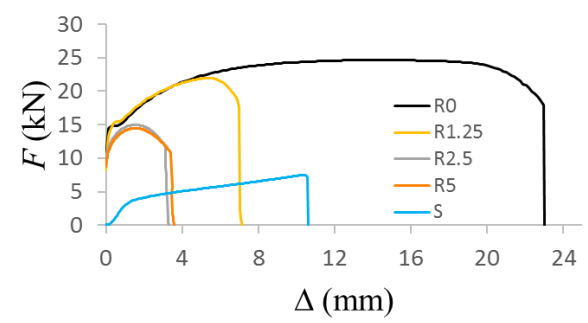

(a)

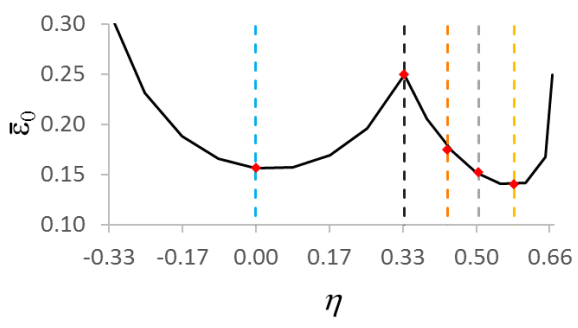

(b)

Figure 1: Experimental results for tensile tests on steel S235: force-elongation curves (a), and (b) ductile damage initiation curve according to the MMC criterion, Eq. (3). R0 indicates tensile experiments on dumb-bell specimens, $\mathrm{R} x$ represents tensile tests on notched samples, where $x$ is the notch radius in $\mathrm{mm}$, and $\mathrm{S}$ denotes the shear response obtained through a tensile experiment, details in Appendix A

Table 1: Values of equivalent plastic strain at necking $\bar{\varepsilon}_{0}$ and stress triaxiality $\eta$ from the experimental tests on steel S235.

\begin{tabular}{lccc}
\hline Test type & $\eta$ & notch radius $(\mathrm{mm})$ & $\bar{\varepsilon}_{0}$ \\
\hline $\mathrm{S}$ & 0 & - & 0.16 \\
$\mathrm{R} 0$ & 0.33 & - & 0.25 \\
$\mathrm{R} 5$ & 0.43 & 5.00 & 0.18 \\
$\mathrm{R} 2.5$ & 0.50 & 2.50 & 0.15 \\
$\mathrm{R} 1.25$ & 0.58 & 1.25 & 0.14 \\
\hline
\end{tabular}

with each geometry, as reported in Table1 The material properties employed in the numerical model were density $\rho=7830 \mathrm{~kg} / \mathrm{m}^{3}$, elastic modulus $E=210 \mathrm{GPa}$ and Poisson's coefficient $\nu=0.33$. Fitting Eq. (3) to the five combinations of $\left(\bar{\varepsilon}_{0}, \eta\right)$ defines the fracture locus plotted in Fig. $1 \mathrm{~b}$, where the best-fit material parameters were obtained as $A=774.9 \mathrm{MPa}, m=0.274, c_{1}=0.033$, $c_{2}=273.6 \mathrm{MPa}$ and $c_{3}=1.016$.

Material strain-rate dependence was implemented by using the CowperSymonds model

$$
\bar{\sigma}(\bar{\varepsilon}, \dot{\bar{\varepsilon}})=\sigma_{0}(\bar{\varepsilon})\left(1+\left|\frac{\dot{\bar{\varepsilon}}}{D}\right|^{\frac{1}{q}}\right),
$$




\subsection{Finite Element model}

Numerical analyses of steel square plates subjected to impulsive loadings were carried out in Abaqus/Explicit to study the failure mechanisms observed experimentally [5, 6]. The experiments involved thin square plates of width $L=89 \mathrm{~mm}$ and thickness $H=1.6 \mathrm{~mm}$, clamped between two rigid frames by means of high-strength bolts. Impulsive blasts were applied by the detonation of explosive strips and the plate response was investigated in a wide range of impulse velocity. 
In the developed FE model, the plate was discretised with a uniform distribution of square S4R shell elements with five integration points through the thickness. Due to the symmetry of the configuration, only one-quarter of the plate was modelled. Fully-clamped boundary conditions were applied along the external edges, while symmetry conditions were defined along the internal edges, as reported in Fig. 2

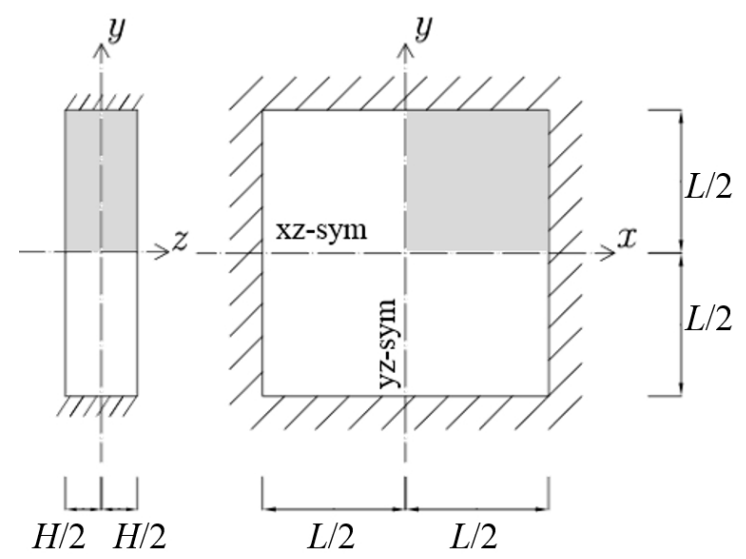

Figure 2: Side (left) and top (right) views of the thin square plate of length $L$ and thickness $H$ subjected to impulsive loads. The external and internal symmetric boundary conditions are highlighted together with the modelled one quarter of the plate (in grey).

Previous studies [5, 14] have shown that the typical blast duration, $15 \mu \mathrm{s}$, is much smaller than the measured response time of $120 \mu \mathrm{s}$. Under these conditions, the loading can be idealised as a zero-period, uniform-momentum impulse, which is modelled through an initial velocity field of intensity $v_{0}$ applied to the plate uniformly [14]. The corresponding impulse intensity, in dimensionless form, is

$$
I^{*}=v_{0} \sqrt{\frac{\rho}{\sigma_{Y}}}
$$

where $\sigma_{Y}$ is the yield stress.

Mesh convergence studies were carried out in two stages. In the first stage, the sensitivity of the damage model to the element size was tested by varying the element size $L_{E}$ in the simulation of the uniaxial tensile tests described in Sec. 2. Fig. 3 shows the comparison between the experimental nominal stress- 

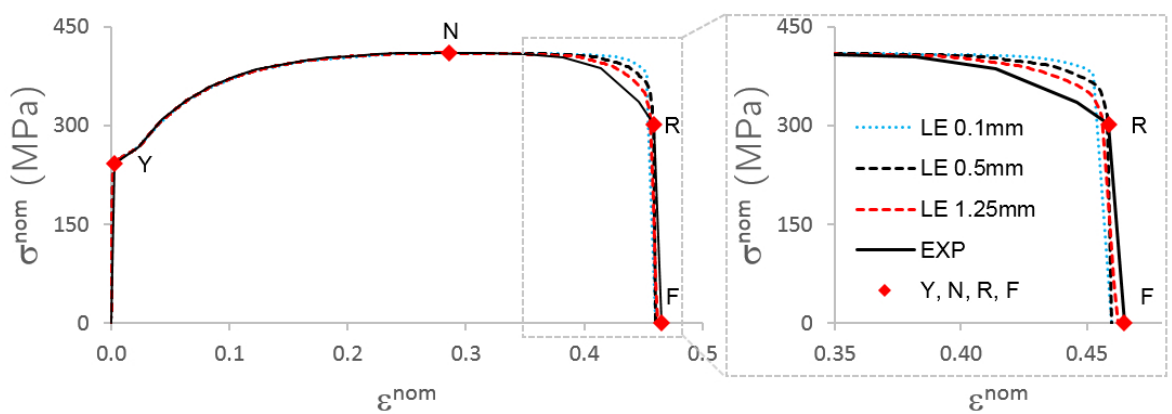

Figure 3: Comparison between the experimental (labelled EXP) nominal stress-strain curve for uniaxial tension on steel S235 and numerical predictions for element sizes $L_{E}=$ $0.1,0.5,1.25 \mathrm{~mm}$. The points of yield, necking, rupture and fracture are highlighted with $\mathrm{Y}, \mathrm{N}, \mathrm{R}$ and $\mathrm{F}$, respectively. The corresponding nominal stress values are $\sigma_{\mathrm{Y}}=242 \mathrm{MPa}$, $\sigma_{\mathrm{N}}=411 \mathrm{MPa}$, and $\sigma_{\mathrm{R}}=302 \mathrm{MPa}$, while the nominal strain values are $\varepsilon_{\mathrm{Y}}=1.15 \cdot 10^{-3}$, $\varepsilon_{\mathrm{N}}=0.29, \varepsilon_{\mathrm{R}}=0.46$ and $\varepsilon_{\mathrm{F}}=0.47$.

strain curve and the numerical results obtained with different element sizes $L_{E}$.

The mesh sensitivity for the damage model is satisfactory in the entire range of $L_{E}$ analysed, as testified by the percentage error on the strain at rupture $\varepsilon_{\mathrm{R}}$, which resulted always smaller than $3 \%$. The second stage was carried out in the simulation of the metal plates, accounting for the strain convergence at the edge mid-length (where strains reach maximum values) and the sensitivity of fracture to the element size. Therefore, the final mesh size was determined as $L_{E}=0.111 \mathrm{~mm}$.

\subsection{Model validation}

The FE model was validated against experimental data for mild steel square plates [5, 6]. The sequence of failure modes previously described was successfully reproduced in the computational model by varying the impulse intensity, as depicted in Fig. 4.

Figure 5 shows the numerical predictions of permanent dimensionless deflection $W / H$ at the plate centre. For mode I, very good agreement is obtained with the experimental data [5, 6] and also with the analytical predictions using a rigid perfectly-plastic plate model (Eq. 23c from Jones [12]). The FE model success- 


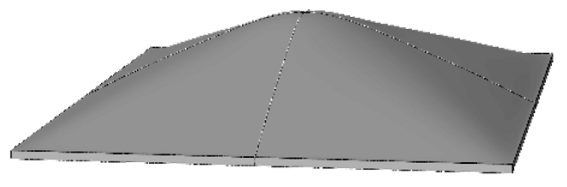

(a) mode I - large inelastic deformation

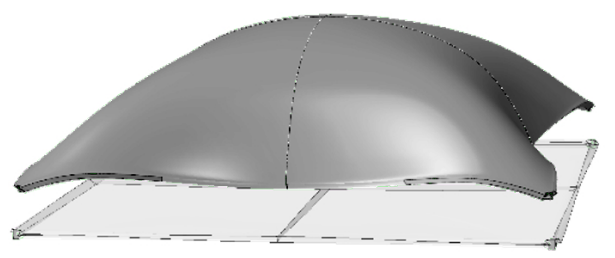

(c) mode II - tensile tearing

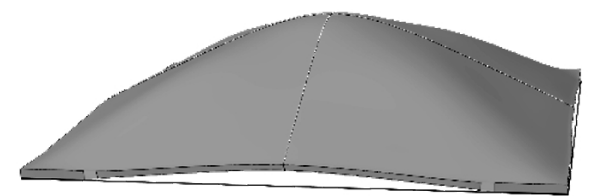

(b) mode II $^{*}$ - partial tearing

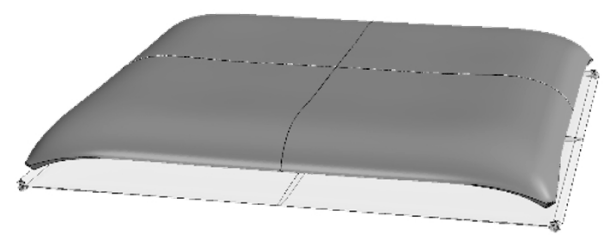

(d) mode III - transverse shear failure

Figure 4: Failure modes of fully-constrained square plates under impulsive loads.

fully predicts a reduction of $W / H$ for mode II with increasing $I^{*}$. In mode II, the discrepancies between the numerically obtained $W / H$ and the experimental measurements can be explained by the different time at which the maximum deflection was calculated, as noticed also by Yuan et al. [14. The maximum deflection in FEM was evaluated at the moment of fracture, whereas the experimental values were obtained a posteriori, after the plate had hit a target. Rudrapatna et al. 13] have previously shown that, after complete detachment, the plate deformation progresses as a result of residual stored kinetic energy and strain, highlighting the importance of the time of measurement of the central deflection. Due to the difference in rupture strain between this study $\left(\varepsilon_{R}=0.46\right.$, steel S235) and the values reported in the experimental studies [5, 6] $\left(\varepsilon_{R}=0.31\right.$, mild steel), an increase in the critical impulse at the mode I-II* transition was observed, as expected.

Numerical predictions of the tear length $T$ in mode II $^{*}$ (i.e. the length of cracks along the plate edge) and of the pull-in displacement $d$ in mode II (i.e. the inward in-plane displacement of the mid-side) are compared with experimental 


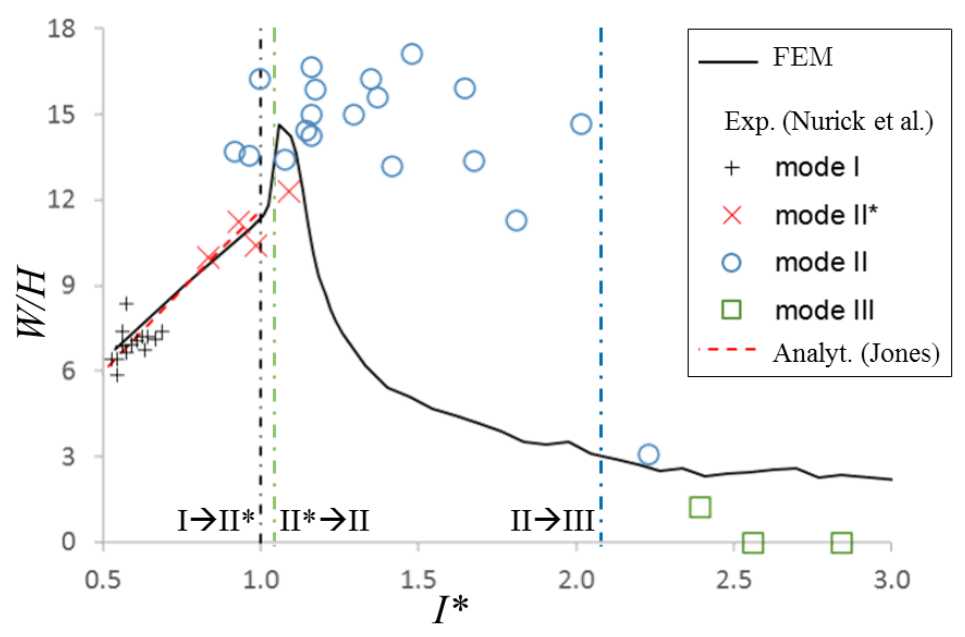

Figure 5: Maximum dimensionless central deflection $W / H$ plotted as a function of dimensionless impulse $I^{*}$. Numerical predictions are compared against experimental data from Nurick et al. 5] 6] (labelled Exp.) and analytical predictions from Jones [12] (labelled Analyt).

data [5, 6] in Table2, It can be concluded that the FE model coherently predicts an initial increase of $d$ with $I^{*}$, followed by a decrease in $d$ at higher impulses, indicated as mode IIa and mode IIb, respectively.

\subsection{Critical impulse at the mode II-III transition}

Experimental observations [5, 6] identified the onset of mode III with a negligible central deflection and a small value of pull-in displacement $(d \leq 1 \mathrm{~mm})$. Rudrapatna et al. [10, 13] suggested that mode II and III are not individually dominated by tensile or shear failure, respectively, but they are the result of an interaction between the two effects, with shear failure becoming predominant as the impulse intensity increases. In particular, in their work it is concluded that the onset of mode III based purely on the amount of deformation is highly subjective, and a more comprehensive criterion must take into account the timeevolution of the failure process, noticing that mode III is also associated with early failure time and instantaneous complete failure [13. This consideration is in agreement with our numerical results, which predict a gradual transition between modes II and III, as evidenced by the slow decrease of the central 
Table 2: Comparison between numerical results and experimental data [5] of the tear length $T$ for mode II $^{*}$, and of pull-in displacement $d$ for mode II.

\begin{tabular}{|c|c|c|c|c|}
\hline & \multicolumn{2}{|r|}{$T_{\mathrm{EXP}}(\mathrm{mm})$} & \multicolumn{2}{|l|}{$T_{\mathrm{FEM}}(\mathrm{mm})$} \\
\hline \multicolumn{2}{|r|}{ Average } & 66 & 61 & \\
\hline \multicolumn{2}{|r|}{ Median } & 61 & 66 & \\
\hline \multicolumn{2}{|r|}{ Maximum } & 80 & 80 & \\
\hline & \multicolumn{2}{|c|}{$d_{\mathrm{EXP}}(\mathrm{mm})$} & \multicolumn{2}{|c|}{$d_{\mathrm{FEM}}(\mathrm{mm})$} \\
\hline & mode IIa & mode IIb & mode IIa $n$ & mode IIb \\
\hline Average & 5.8 & 3.5 & 5.4 & 2.9 \\
\hline Median & 6.0 & 3.5 & 5.4 & 3.1 \\
\hline
\end{tabular}

deflection at high impulses in Fig. 5. In light of these observations, a more rigorous definition of the critical impulse at the mode II-III transition is necessary, based on quantities that are directly related to the failure mechanisms. Suitable quantities are the tear length $T$ and the crack propagation velocities along the supports. In our FE results, $T$ varies in the small range of $95 \div 98 \%$ of $L$ in both failure modes for all values of $I^{*}$. However, it is observed that the crack propagation velocity increases monotonically with $I^{*}$ in the range of impulses considered, varying from subsonic to supersonic values.

Therefore, we propose a phenomenological criterion for determining the critical value $I_{I I-I I I}^{*}$ based on the definition of the dimensionless critical crack speed

$$
v^{*}=\frac{v_{\mathrm{crack}}}{c}
$$

where

$$
c=\sqrt{\frac{E(1-\nu)}{\rho(1+\nu)(1-\nu)}}
$$

is the propagation velocity of pressure waves in solids. When $v^{*} \geq 1$, the plate response is categorised as mode III. The proposed criterion satisfies the conditions on both $d$ and $W / H$. The corresponding predicted critical impulse for the analysed square plate, $I_{I I-I I I, \mathrm{FEM}}^{*}=2.10$, results in close agreement 
with the experimental measurement, $I_{I I-I I I, \mathrm{EXP}}^{*}=2.39[5]$.

\section{Results and Discussions}

The typical distribution of stress triaxiality $\eta$ through a plate deforming in mode II $\left(I^{*}=1.08\right)$ is displayed in Fig. 6. Rupturing always initiates simultane-

210 and Fig. 6b. Subsequently, the cracks rapidly propagate through most of the side; as time increases, the crack tips approach the corner, and the region under equibiaxial tension reduces (Fig. 6c). The instants immediately before and after complete detachment are displayed in Fig. 6 d, e and f, showing that the

\subsection{Effect of plate thickness and area}

Two parametric studies were performed to assess the effect of the plate thickness $H$ and volume $V$ on the inelastic response. In the first study, the 


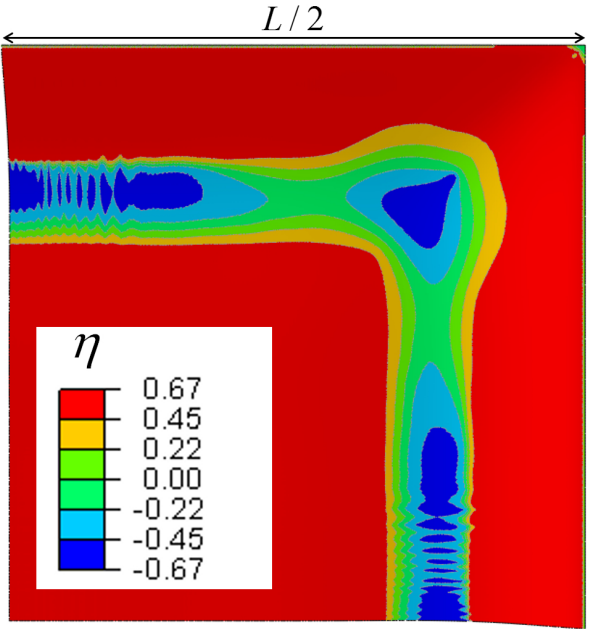

(a) Prior to rupture, $t=1.8 \mathrm{e}-5 \mathrm{~s}$

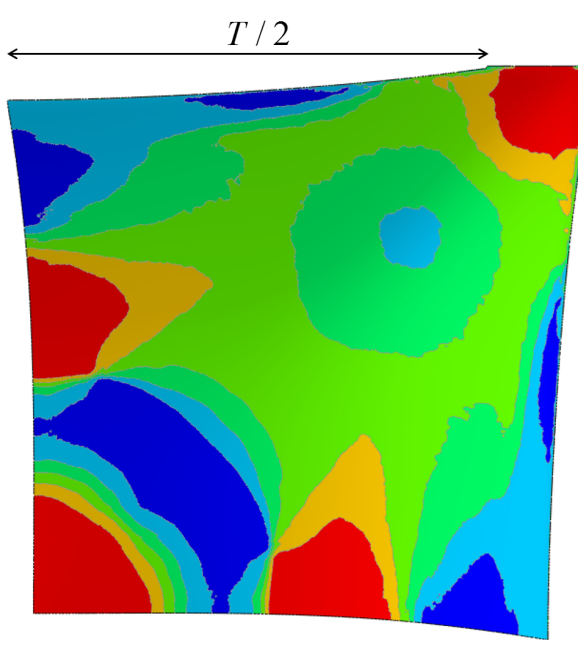

(c) Propagation, $t=7.30 \mathrm{e}-5 \mathrm{~s}$

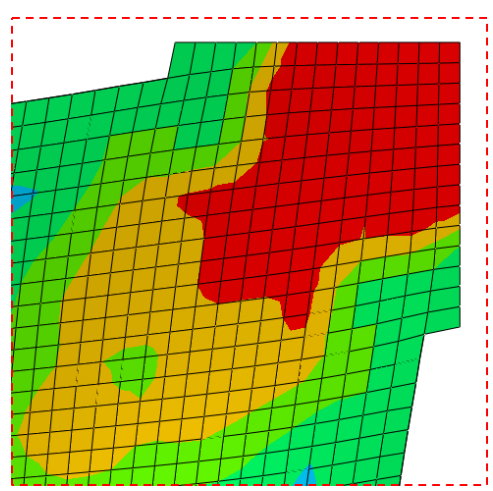

(e) Deviation (detail)

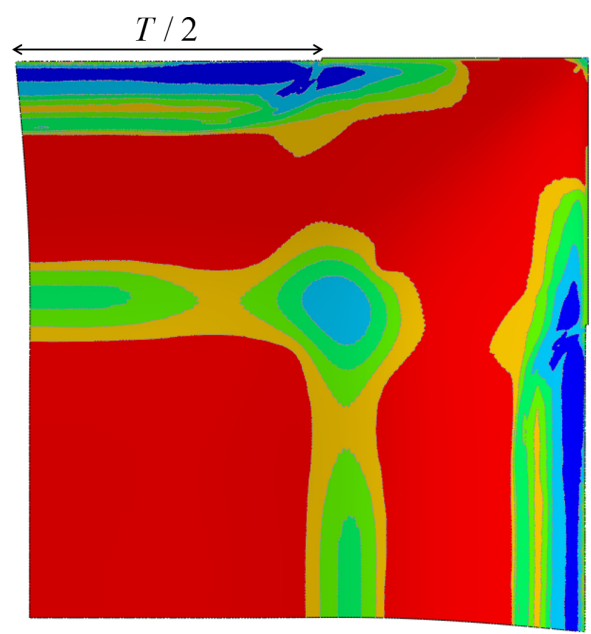

(b) Rupture, $t=3.4 \mathrm{e}-5 \mathrm{~s}$

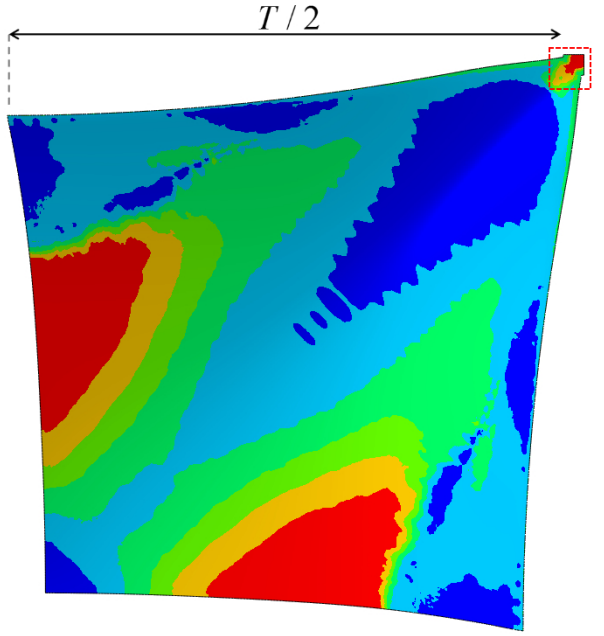

(d) Deviation, $t=11.2 \mathrm{e}-5 \mathrm{~s}$

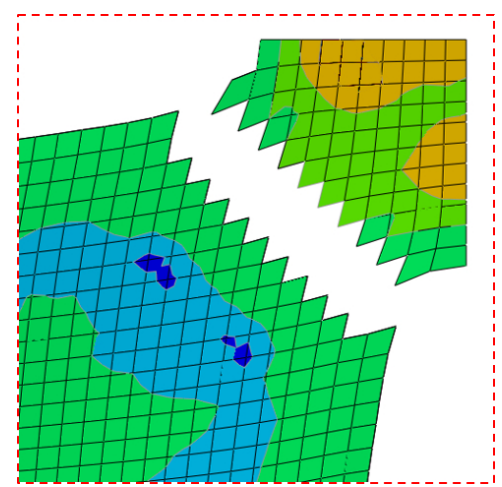

14

(f) Detachment, $t=11.4 \mathrm{e}-5 \mathrm{~s}$

Figure 6: Time evolution of the stress triaxiality $\eta$ distribution and of the tear length $T$ for a square plate in mode II, subjected to a dimensionless impulse $I^{*}=1.08$. The images report the plate top view from the side of the incident blast. 
thickness was varied in the range $H=1.6 \div 6 \mathrm{~mm}$ whilst keeping the width constant $(L=89 \mathrm{~mm})$ whereas, in the second study, $H$ was varied in the same range whilst keeping the volume constant. The study was repeated for several values of volume in the range $V=12674 \div 47526 \mathrm{~mm}^{3}$. According to the Kirchoff-Love theory, the aspect ratio $L / H$ between the plate width and values of the ratio were selected in the range $L / H=15 \div 56$

The results of the first parametric study are presented in Fig. 7. For all the cases analysed, deviation from linearity between $W / H$ and $I^{*}$ always corresponds to the initiation of mode $\mathrm{II}^{*}$, whereas the maximum central deflection corresponds to the initiation of mode II. As expected, increasing $H$ significantly reduces the plate's deformability, and lower deflections are observed. This effect is more evident for thick plates $(L / H \leq 20)$, with a pronounced flattening of the peak of $W / H$.

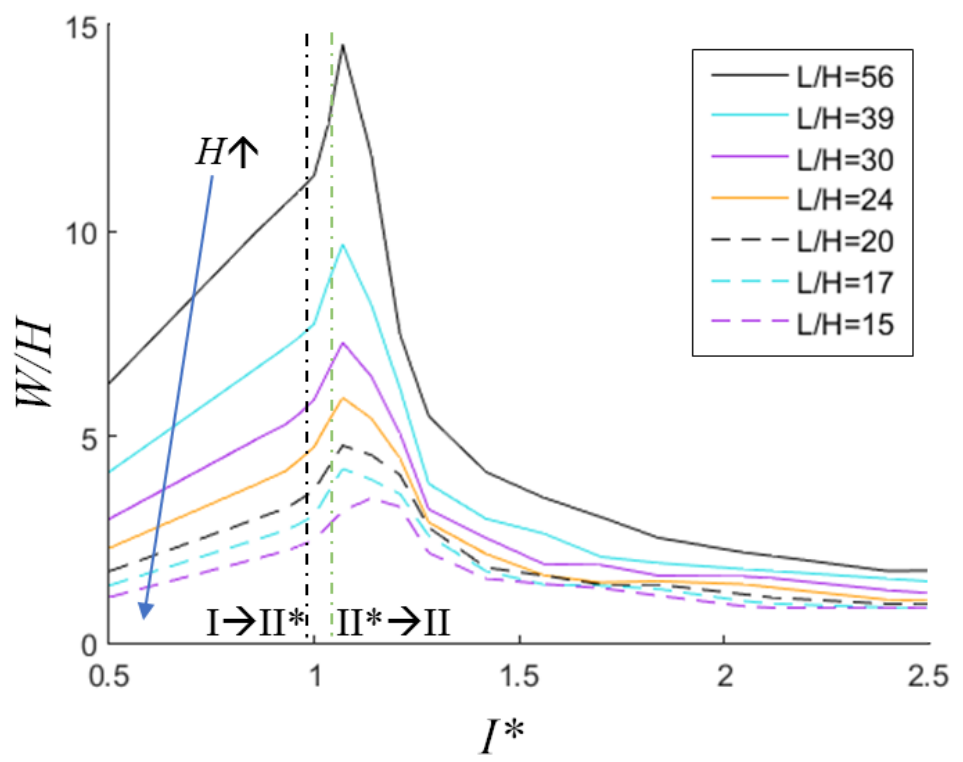

Figure 7: Numerical predictions of $W / H$ for the first parametric study in which the thickness $H$ ranges between 1.6 and $6 \mathrm{~mm}$ while the length $L$ remains constant at $89 \mathrm{~mm}$.

Dimensionless diagrams, named failure maps, are introduced in Fig. 8 to 
mechanisms only depend on the amount of strain accumulating along the support. On the contrary, the region of mode III becomes smaller because, as the thickness increases, the plate is capable of absorbing more energy against the propagation of the cracks, and the propagation speed decreases. The results of Fig. 7 are then overlaid on the failure map in Fig. 8 a as isolines of $W / H$.

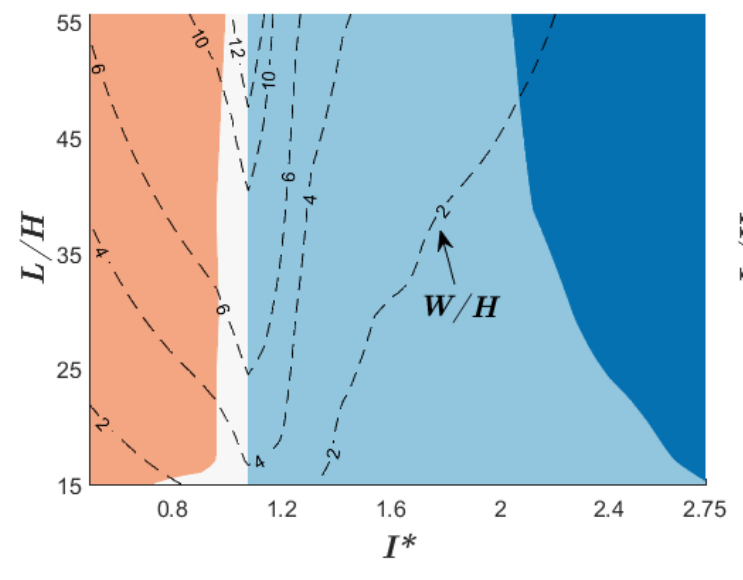

(a)

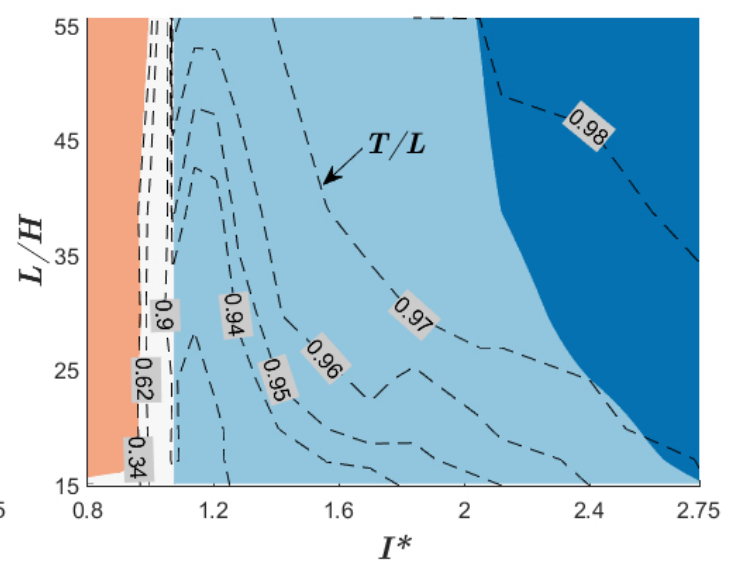

(b)

Figure 8: Failure maps for the first parametric study $(H=1.6 \div 6 \mathrm{~mm}, L=89 \mathrm{~mm})$ displaying the boundaries between failure modes, overlaid with isolines of $W / H$ (a) and of $T / L$ (b). Colours are used to identify failure mode I $\square$, mode II $\square$, mode II $\square$, and mode III $\square$.

Failure maps displaying the effect of the geometry on the tear length, as isolines of $T / L$, are shown in Fig. $8 \mathrm{~b}$ and Fig. 9 for the first and second parametric study, respectively. It can be seen that there is an almost perfect correspondence between the modes $\mathrm{I}_{-} \mathrm{II}^{*}$ transition and the isoline $T / L=0.34$, indicating that 265 the minimum tear length is independent of $H$ and $V$. In the narrow range of 
impulses where mode $\mathrm{II}^{*}$ occurs, $T / L$ varies from 0.34 to 0.90 . The implication is that small increments in the impulse intensity correspond to large variations of the tear length for mode $\mathrm{II}^{*}$. It is observed that the isoline $T / L=0.98$ passes through the mode III region, indicating that the crack deviation occurs even at high impulses, and a small amount of material remains connected to the support. Numerical predictions have been compared in the previous section with experimental data for a plate of thickness $H=1.6 \mathrm{~mm}$ [5, 6]. Further comparisons against analogous data for plates of different thickness are required for the complete validation of the model and the failure maps, which could then be proposed for the use in plate design.

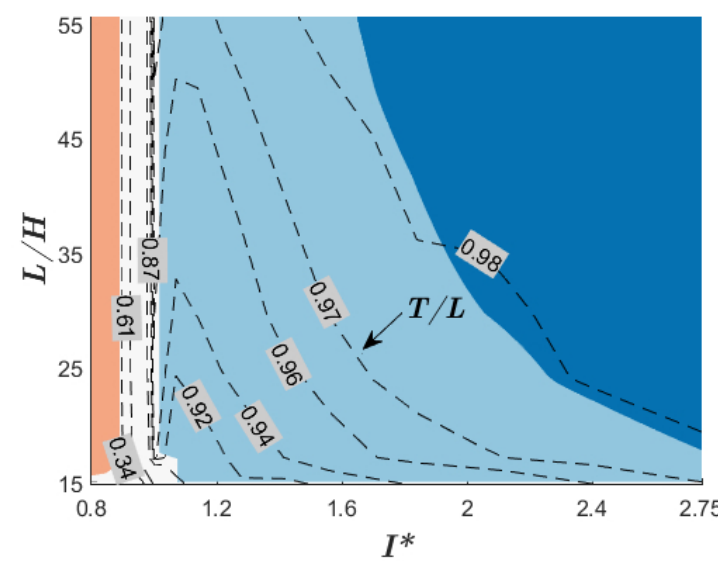

(a) $V=12674 \mathrm{~mm}^{3}$

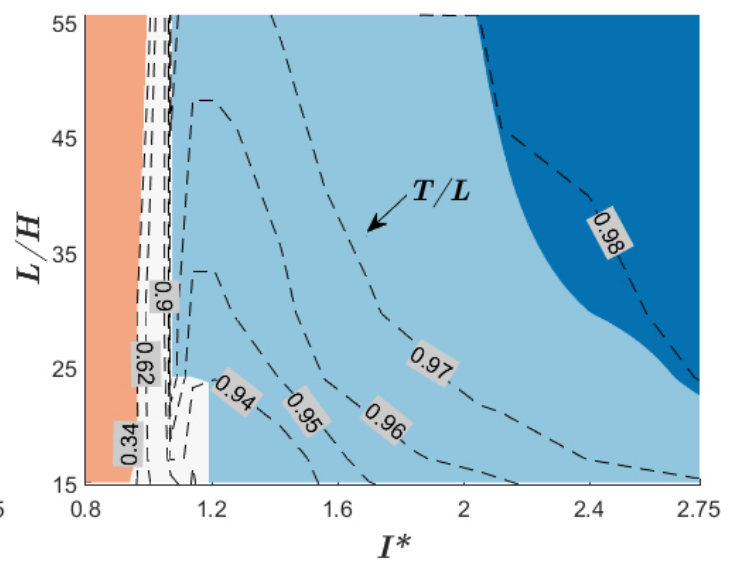

(b) $V=47526 \mathrm{~mm}^{3}$

Figure 9: Failure maps of predicted $T / L$ from the second parametric study, in which $H=$ $1.6 \div 6 \mathrm{~mm}, V=12674 \mathrm{~mm}^{3}$ (a) and $V=47526 \mathrm{~mm}^{3}$ (b). Colours are used to identify failure mode I $\square$, mode II* $\square$, mode II $\square$, and mode III 


\subsection{Failure of rectangular plates}

A numerical study was conducted to analyse the response of fully clamped rectangular plates under impulsive loading conditions. The plates have the same thickness $H=1.6 \mathrm{~mm}$ and surface area $A=7921 \mathrm{~mm}^{2}$ of the square plates analysed in Sec. 3. The aspect ratio $\gamma$ between the longer and the shorter side was varied in the range $\gamma=L_{A} / L_{B}=1 \div 5$, Fig. 10 .

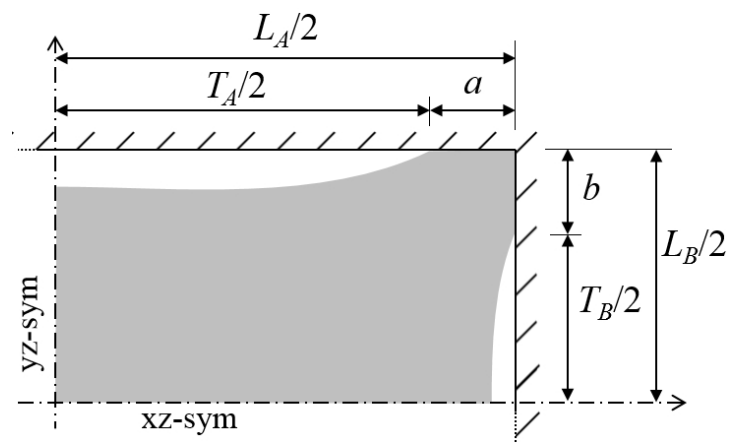

Figure 10: Geometry of one quarter of a rectangular plate with fully-constrained boundary conditions. The grey area highlights the post-failure configuration prior to detachment. The tear lengths on the longer and shorter sides are indicated with $T_{A}$ and $T_{B}$, respectively.

The simulated rectangular plates display the same failure modes as the square plates. The only difference is observed in mode II $^{*}$, where at low impulses failure involves only the longer edge, where crack first propagates. When failure modes II or III occur, the crack propagation velocity is initially faster on $L_{A}$ compared to $L_{B}$ until the residual length on the long edge matches that on the short edge, $a \approx b$. Afterwards, the two cracks propagate with the same speed until detachment occurs. The crack deviation follows a diagonal crack path with $a / b \approx 1$ for all combinations of $\gamma$ and $I^{*}$ studied. In the case of square plates, $a$ and $b$ were equal because of the symmetry of the geometry. The reason why $a \approx b$ for rectangular plates is found by looking at the distribution of $\bar{\varepsilon}$ and $\eta$ before fracture. It can be seen from Fig. 11 that, because of the mode of deformation of rectangular plates [27, 28, the distribution's axis of symmetry does not follow the plate diagonal, but a line inclined at $45^{\circ}$ from the plate corner. 


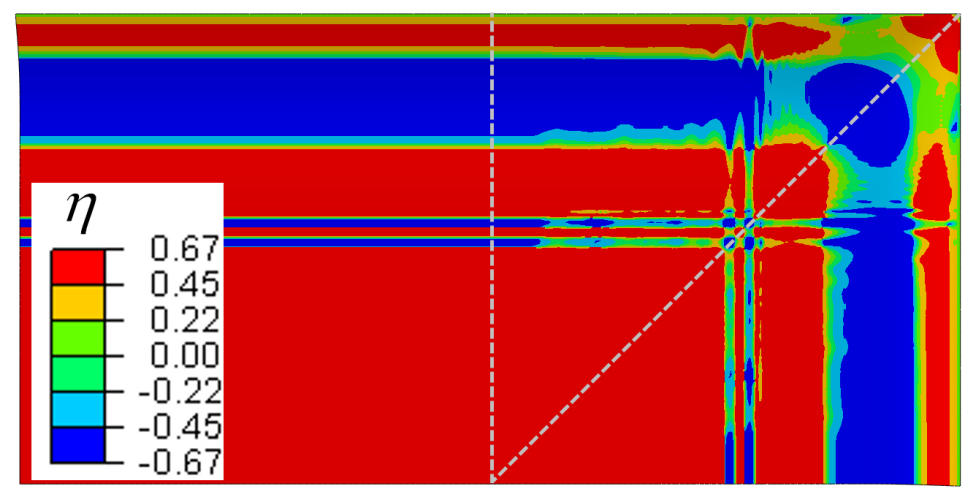

(a) Prior to rupture, $t=0.4 \mathrm{e}-5 \mathrm{~s}$

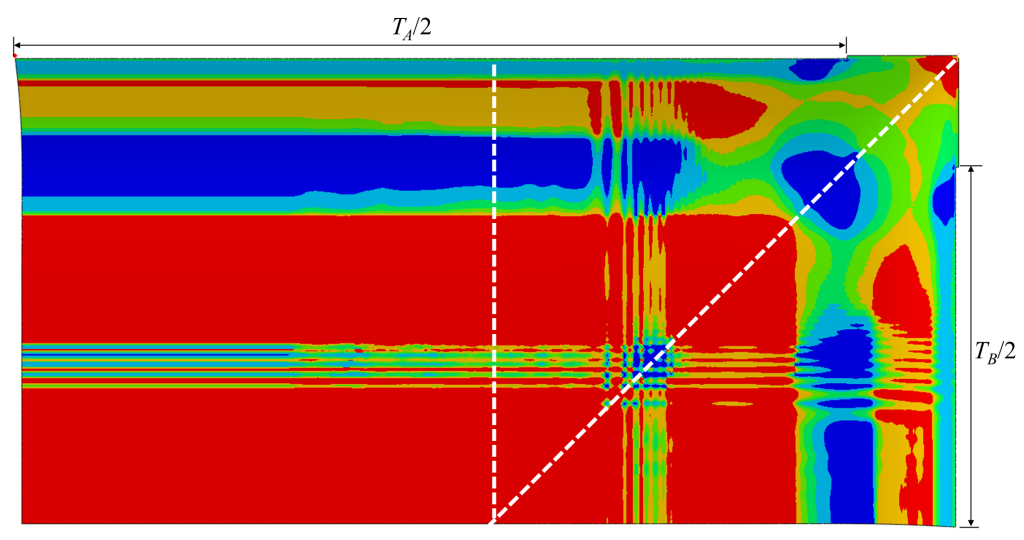

(b) Rupture, $t=0.8 \mathrm{e}-5 \mathrm{~s}$

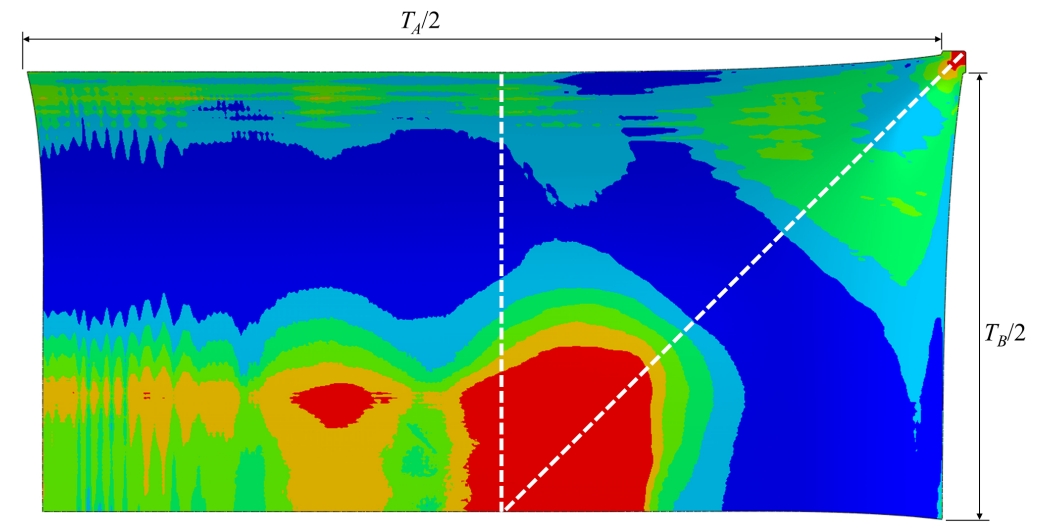

(c) Detachment, $t=2.8 \mathrm{e}-5 \mathrm{~s}$

Figure 11: Time evolution of stress triaxiality $\eta$ distribution for a rectangular plate with $\gamma=2$ deforming in mode II when subjected to a dimensionless impulse $I^{*}=2.7$. 

rapid decrease of $W / H$, and the plates tend to the same amount of deflection, regardless of the aspect ratio, for higher impulses.

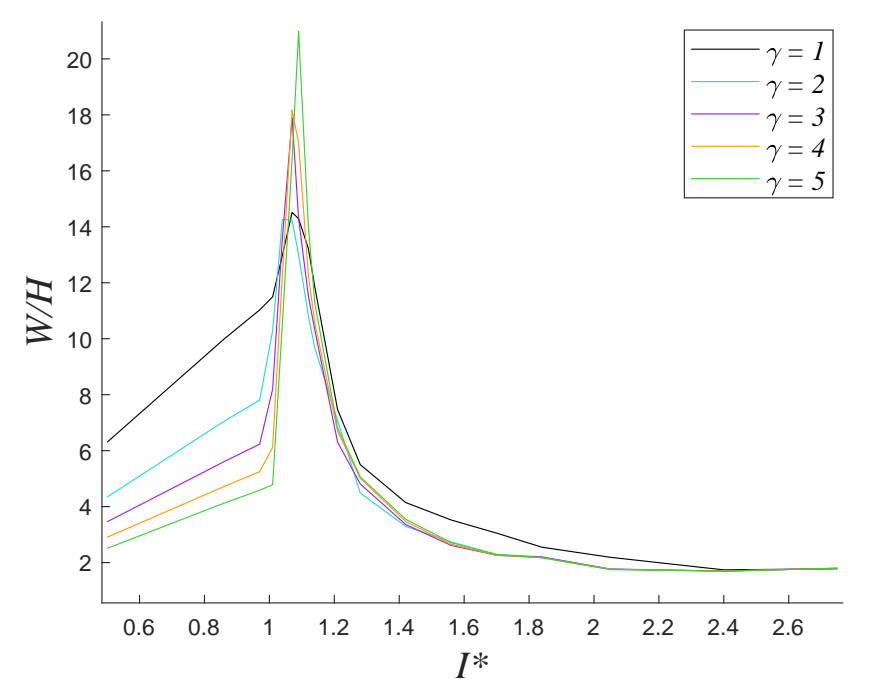

Figure 12: Numerical predictions of $W / H$ as a function of dimensionless impulse $I^{*}$ for rectangular plates with fully-constrained boundary conditions and different aspect ratio $\gamma$.

The boundaries between failure modes are displayed in the failure map of Fig. 13a. The aspect ratio has a negligible effect on the mode ${\mathrm{I}-\mathrm{II}^{*}}^{*}$ and $\mathrm{II}^{*}$-II boundaries, and mode $\mathrm{II}^{*}$ only occurs in a low range of impulses. Furthermore, the aspect ratio shows no effect on the measured time to rupture and time to detachment, therefore the crack propagation velocity increases with $\gamma$, which corresponds to an increase in the extent of the mode III region.

Fig. 13b shows the failure map of the residual length on the long edge, $T_{A}$,

adimensionalised by $L_{A}$. For all instances where detachment occurs, $T_{A} / L_{A}>0.93$ 
and the residual length is negligible on both edges. Then, since $T_{A} \approx L_{A}$ and $T_{B} \approx L_{B}$, it is found that $T_{A} / T_{B} \approx \gamma$.

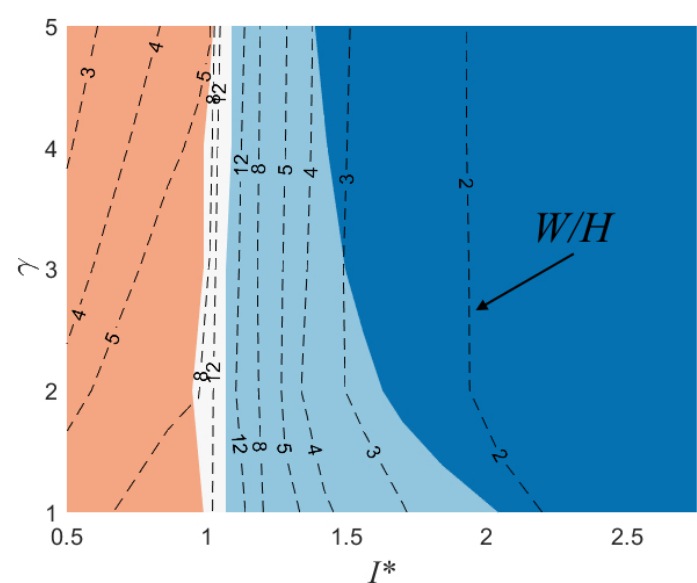

(a)

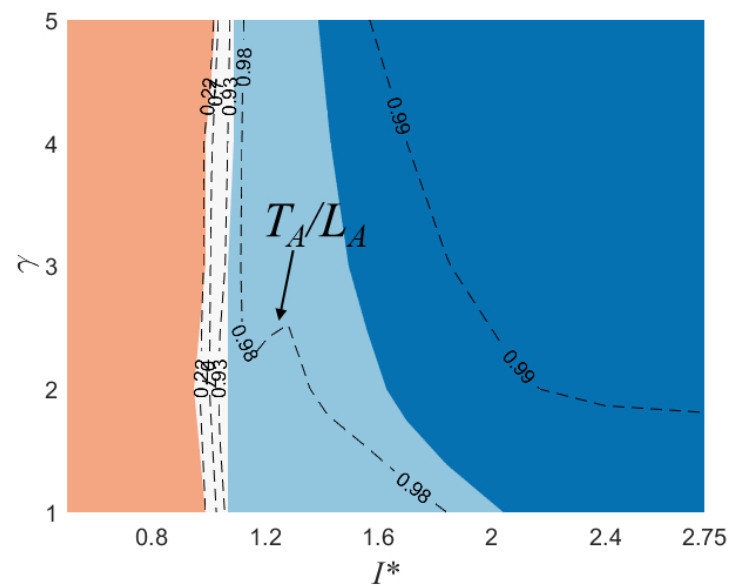

(b)

Figure 13: Failure map of predicted $W / H$ (a) and of $T_{A} / L_{A}$ (b) for fully-constrained rectangular plates with different aspect ratio $\gamma$. Colours are used to identify failure mode I $\square$, mode $\mathrm{II}^{*} \square$, mode II $\square$, and mode III

\subsection{Failure of simply-supported square and rectangular plates}

Previous studies demonstrated that the failure modes can be affected significantly by changes in the boundary conditions [3]. For instance, Bonorchis et al. [7] and Thomas [9] showed that plates with built-in or welded boundaries incur into earlier failure, compared to fully-clamped plates, due to the increased rigidity of the boundary, which limits the in-plane displacement of the plate at its supports. In the literature, little attention has been given to the case of less rigid boundary conditions. For this reason, numerical studies were conducted to analyse the response of impulsively loaded metal plates with simply-supported boundary conditions, when the rotation of the plate at the supports is allowed.

It is expected that the additional rotational degrees of freedom affect the sequence of failure modes of quadrangular plates, influencing the impulse range for each mode, the amount of deformation, and the position of rupture. Starting 
from mode I, Figs. 14a and 14b compare the deformation profiles of square plates with different boundary conditions. In the case of fully-constrained boundary conditions, the profile resembles a uniform dome shape, whereas a more complex profile is observed for simply-supported boundary conditions, with a small central dome superimposed atop the global dome, joined by an inflection point. This behaviour resembles that observed experimentally for plates subjected to localised blast loadings [2, 3, 8, 11] which are characterised by a similar profile, as a consequence of a non-uniformly distributed velocity field.

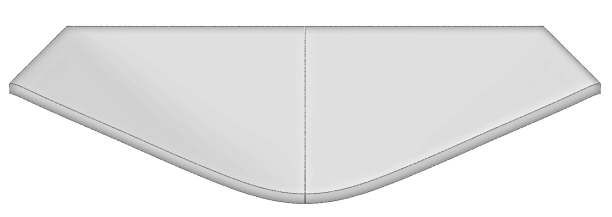

(a)

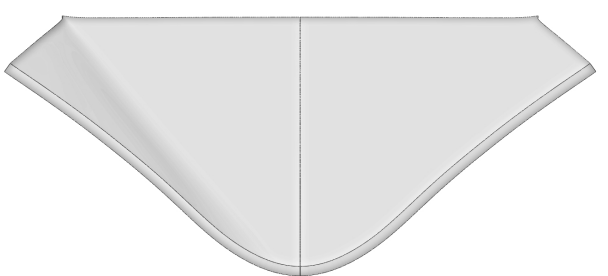

(b)

Figure 14: Deformation profile of square plates subjected to blast loadings deforming in mode I: (a) fully-constrained boundary conditions and (b) simply-supported boundary conditions.

The other failure modes found for simply-supported square plates are depicted in Fig. 15. Increasing the impulse intensity, mode I is followed by a failure mode, different from the mode $\mathrm{II}^{*}$ of fully clamped plates, characterised by partial tearing in the central region, labelled as mode $\mathrm{II}^{*} \mathrm{c}$. This is then followed by complete tearing in the central area, labelled as mode IIc, a response also known as 'capping' [3], which is normally observed for locally loaded plates. The amount of deformation and the capping area grow with the impulse intensity. Increasing the impulse further, complete tearing occurs along crack paths that run parallel to the plate edges, resembling failure mode II and III, respectively.

A different sequence of failure modes was observed for simply-supported rectangular plates $(\gamma=2 \div 5)$, as displayed in Fig. 16. By increasing the impulse intensity, mode I is followed by mode II*A, where partial tearing occurs 


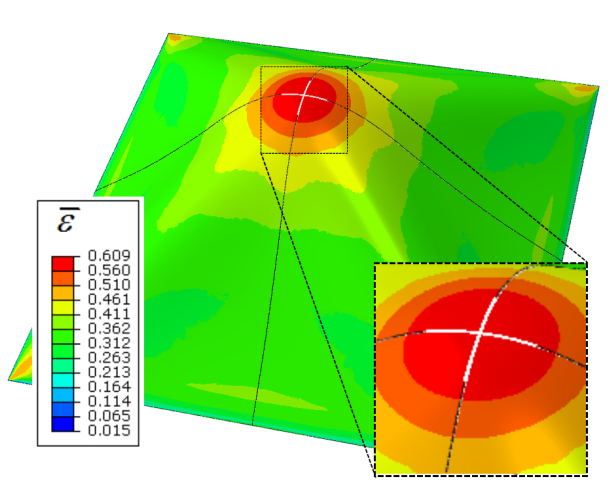

(a) mode II $^{*} \mathrm{c}$ - partial central tearing

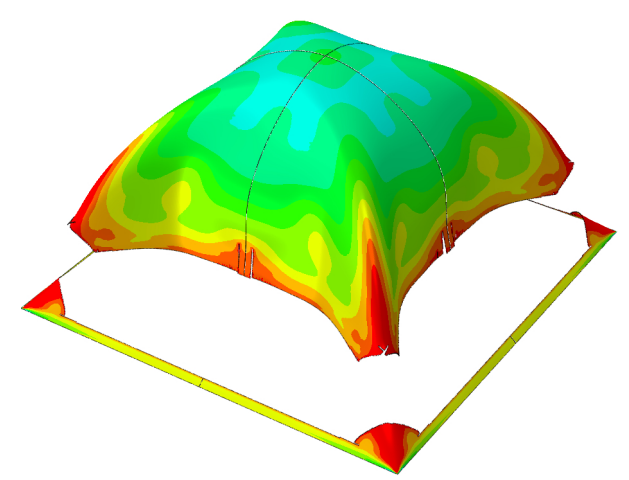

(c) mode II

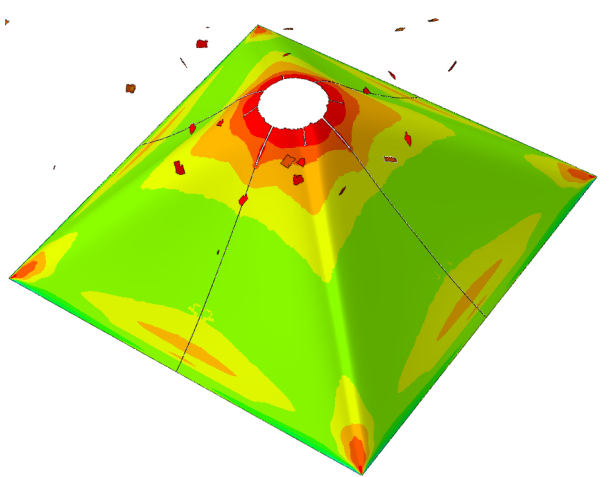

(b) mode IIc - capping

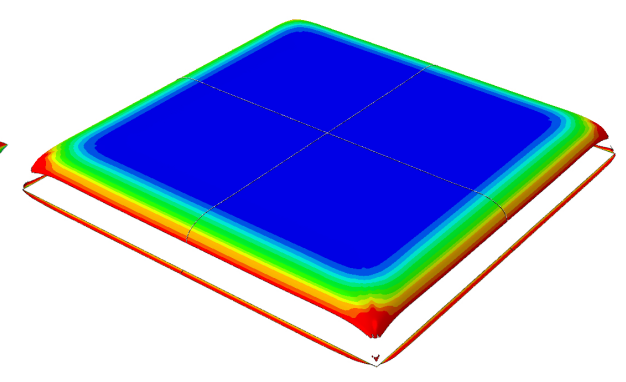

(d) mode III

Figure 15: Failure modes of simply-supported square plates. Contour plots of equivalent plastic strain $\bar{\varepsilon}$ are used to highlight regions of maximum strain, where failure occurs.

on a path running parallel to the long edge, but not on the short edge. At higher impulses the rupture mechanism deviates from its path with a sharp angle and propagates towards the opposite edge. Complete failure occurs when two crack path from opposite edges meet, causing the detachment of a large section of the plate. To the best of the authors' knowledge, this failure mode has not been described previously in the literature and will be here referred to as mode IIr or 'ripping'. This mode can be partial or complete, depending on whether the crack stops in its propagation, and is followed at higher impulses by modes II and III. 


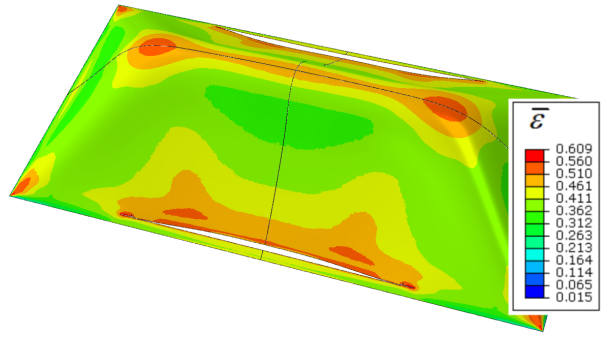

(a) mode II*A - partial tearing along long edge

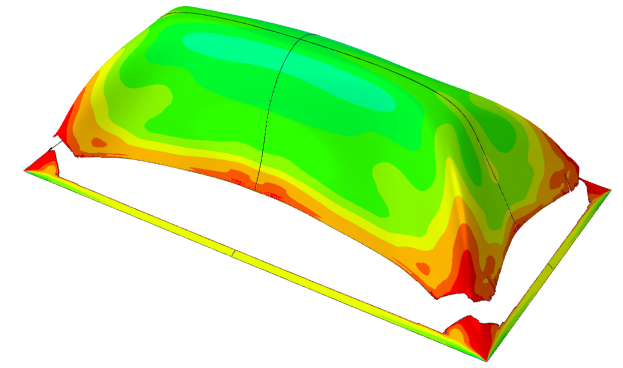

(c) mode II

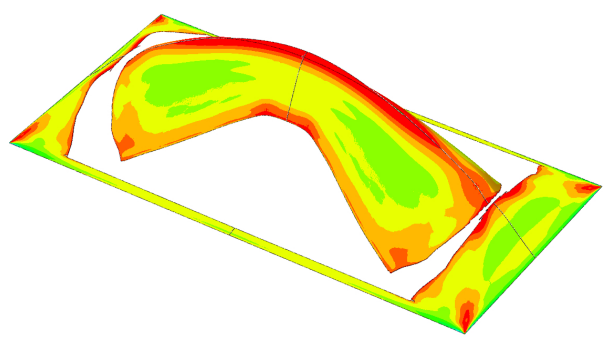

(b) mode IIr - ripping

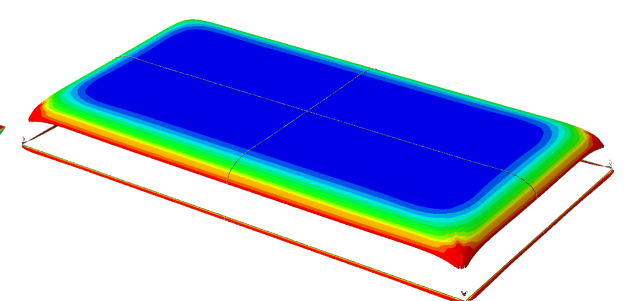

(d) mode III

Figure 16: Failure modes of simply-supported rectangular plates with $\gamma=2$. Contour plots of equivalent plastic strain $\bar{\varepsilon}$ are used to highlight regions of maximum strain, where failure occurs.

It is observed that the tearing distance from the support is negligibly affected by the aspect ratio $\gamma$ and the failure mode. One distinguishing feature of the detachment path for simply-supported plates consists in the multiple branches that form along the tears, compared to the straight fracture without branches that characterises the fully-constrained plates.

The measured dimensionless central deflection $\mathrm{W} / \mathrm{H}$ and the sequences of failure modes are reported in Fig. 17. It is observed that the increased mobility of the boundaries reduces the strain in the peripheral regions, and mode I occurs in a wider range of impulses. Consistently with previous studies, deviation from linearity coincides with the initiation of rupture mechanisms: mode $\mathrm{II}^{*} \mathrm{c}$ for square plates (Fig. 17a), and mode II*A for rectangular plates (Fig. 17b). Peak values of deflection are always reached at the onset of mode II, followed by a 


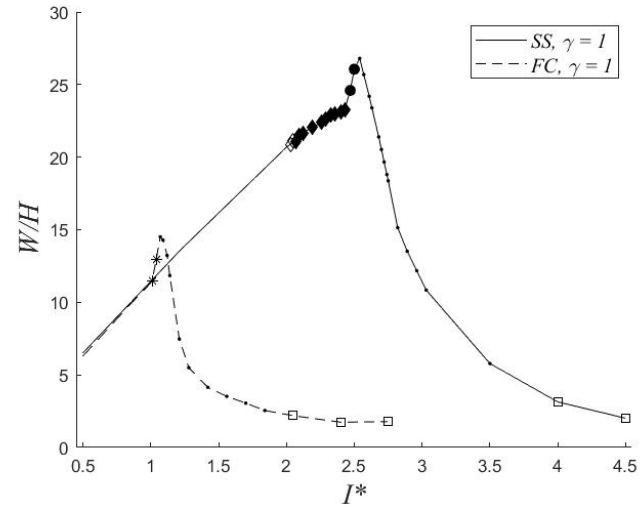

(a)

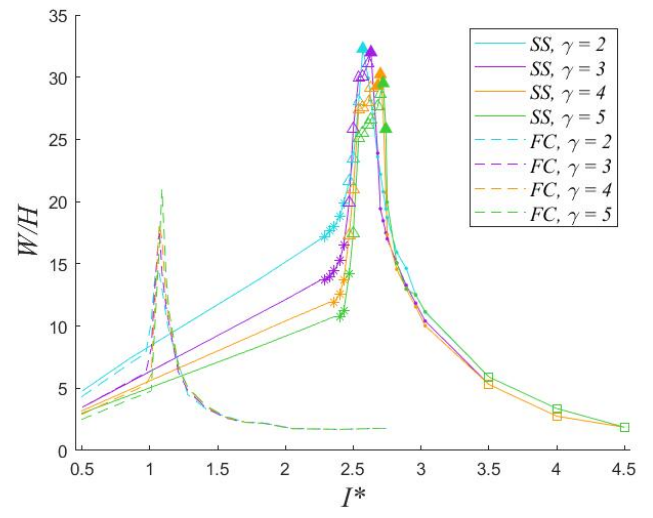

(b)

Figure 17: Comparison of dimensionless deflection $W / H$ for plates with simply supported (SS) or fully-constrained (FC) boundary conditions: (a) square plates and (b) rectangular plates. Symbols are used to highlight failure modes as follows: * mode $\mathrm{II}^{*}$ and mode $\mathrm{II}^{*} \mathrm{~A}$, $\diamond$ mode II $^{*}$ c $\diamond$ mode IIc - capping, $\bullet$ interposition of capping and mode II, • mode II, $\square$ mode III, and mode IIr with $\triangle$ partial ripping and $\boldsymbol{\Delta}$ complete ripping.

rapid drop at higher impulses. Based on the proposed criterion on crack propagation velocity, the transition between mode II and III occurs at impulses higher than $I^{*}=3.5$, although the horizontal asymptote typical of fully-constrained plates was not observed in the range of impulses analysed.

The measurements of tear lengths $T_{A}$ for rectangular plates from this study is reported in the failure map of Fig. 18 Analogously to the fully constrained plates, the greatest variation of $T_{A}$ occurs in the impulse range of mode $\mathrm{II}^{*}$, and increases slowly with the impulse in the ranges of mode II and III. Similarly to 375 fully clamped plates, we observed that $a \approx b$ and $T_{A} / T_{B} \approx \gamma$, for any value of $\gamma$ and $I^{*}$.

\section{Conclusions}

A finite element model has been presented for the prediction of the inelastic response of quadrangular steel S235 plates subjected to impulsive blast loadings. The developed model incorporates comprehensive failure criteria, based 


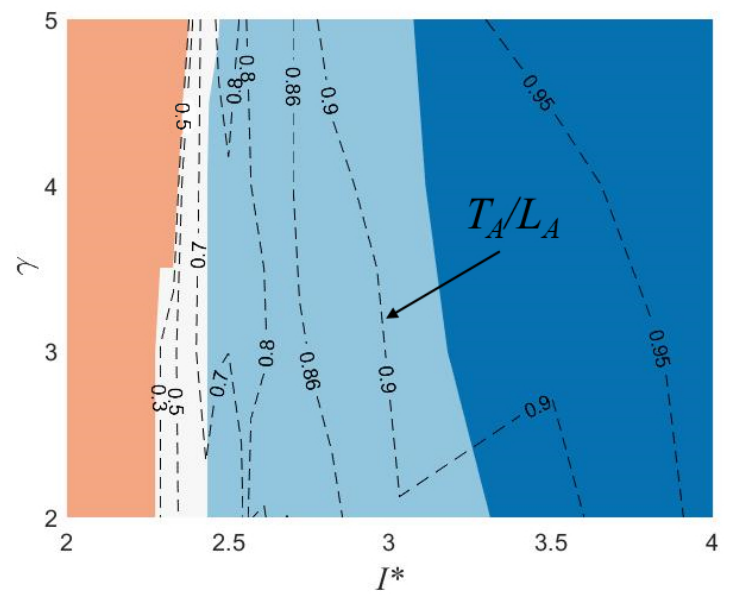

Figure 18: Dimensionless tear length $T_{A} / L_{A}$ failure map for simply-supported rectangular plates. Colours are used to identify failure mode I $\square$, mode II*A $\square$, mode II and IIr $\square$, and mode III

on a continuum damage mechanics approach, calibrated on experimental tensile and shear tests. The numerical results have been compared with available experimental data of central deflection, tear length and pull-in displacement for thin square plates [5, 6, showing a good agreement. Furthermore, a novel criterion to assess the onset of failure mode III has been proposed, based on the crack propagation speed.

The established numerical model has been employed in the prediction of the dynamic response of quadrangular plates with fully-constrained or simplysupported boundary conditions. The new results indicate that simply-supported plates subjected to uniform blast loading display failure modes different from those of fully-clamped panels. The observed behaviour is similar to that of plates experiencing localised blast, with the development of a non-uniform deformation profile and 'capping' failure. In addition, a previously unobserved 'ripping' failure mode has been identified for simply-supported rectangular plates, where detachment occurs due to the joining of crack paths propagating from opposite sides, rather than from contiguous edges.

Finally, dimensionless failure maps have been developed, elucidating the 
influence of plate topology, boundary conditions and amount of material on the inelastic response. The maps represent a valid aid in the design of impulsively loaded plates since they indicate the failure modes, tear and residual lengths, and the maximum expected deformation as a function of the impulse intensity.

\section{Acknowledgements}

The last author wishes to acknowledge the funding awarded by QinetiQUK (Mr Robert Ball - Structures \& Survivability, Platform Design and Life Support IDT) which was used to support the first author in the form of a $\mathrm{PhD}$ studentship.

\section{Appendix A. Experimental characterisation of damage criterion}

A series of experiments were required to identify the fracture locus defined by the MMC damage initiation criterion of Eq. (3). The first test was a uniaxial experiment, carried out to obtain the strength coefficient $A$, the strain hardening exponent $n$, and the value of strain at necking $\bar{\varepsilon}_{0}(\eta=1 / 3)$. The calibration of the remaining parameters, namely $c_{1}, c_{2}, c_{3}$, can be fulfilled by fitting Eq. (3) to experimental data of $\bar{\varepsilon}_{0}$ for other values of stress triaxiality $\eta$. Brünig et al. 24] and Driemeier et al. 25] showed that different values of $\eta$ can be obtained ${ }_{415}$ in notched dumb-bell specimens loaded in uniaxial tension. Fig. A.19 depicts the coupon geometries used in this study: R0 indicates a typical prismatic dogbone specimen, R1.25, R2.5 and R5 indicate prismatic specimens with a central notch of radius $R=1.25,2.5,5 \mathrm{~mm}$, respectively [29]. Depending on the radius, values of triaxiality $\eta=0.58,0.50,0.43$ were obtained in the central region of the specimen, where necking occurs. The last specimen, S, presents a different geometry that allows to obtain pure shear conditions in the central region at the onset of fracture. 


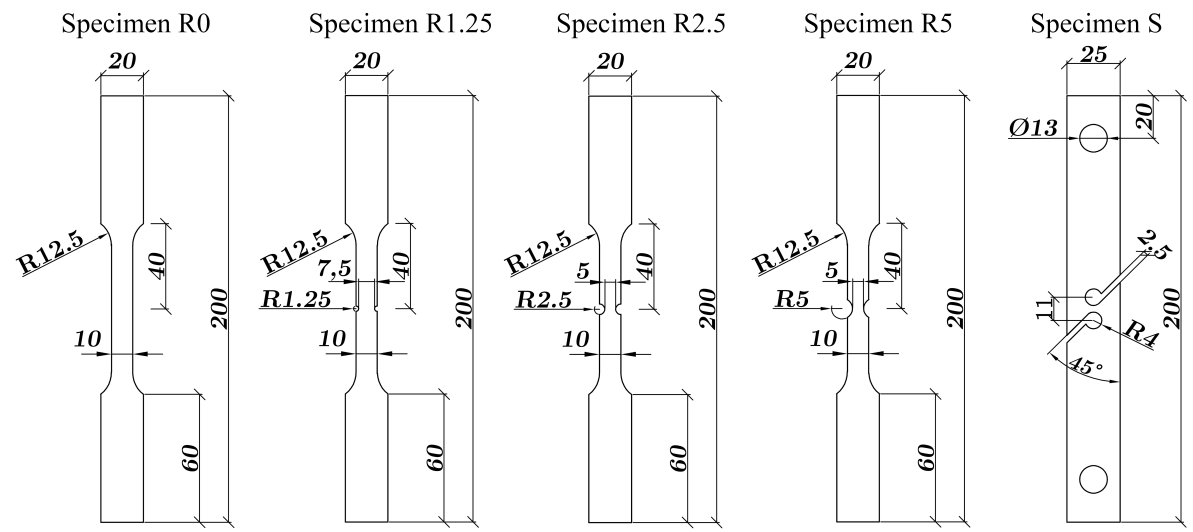

Figure A.19: Geometries of un-notched (R0), pre-notched (R1.25, R2.5 and R5), and shear (S) specimens. The specimens have thickness $6 \mathrm{~mm}$ and initial gauge length of $50 \mathrm{~mm}$. All dimensions are in $\mathrm{mm}$.

\section{Appendix B. Definition of the damage variables $D_{D}$ and $D_{S}$}

The loss of load-carrying capacity of the material after necking is modelled following the continuum damage mechanics approach through the damage variable $D_{D}$, evaluated from uniaxial tensile test data using the relationships [21]

$$
D_{D}(\bar{\varepsilon})= \begin{cases}1.5\left(1-\frac{\sigma_{0}(\bar{\varepsilon})}{\sigma^{\prime}}\right) & \text { before rupture } \\ 1 & \text { after rupture }\end{cases}
$$

with

$$
\sigma^{\prime}= \begin{cases}\sigma^{\text {nom }}\left(1+\varepsilon^{\text {nom }}\right) & \text { before necking } \\ \sigma_{\mathrm{N}}^{\text {nom }}\left(1+\varepsilon^{\text {nom }}\right) & \text { after necking }\end{cases}
$$

where $\varepsilon^{\text {nom }}$ and $\sigma^{\text {nom }}$ are the nominal strain and stress, respectively, $\sigma_{\mathrm{N}}^{\text {nom }}=$

$411 \mathrm{MPa}$ is the value of stress at necking, as reported in Fig. 3 and $\sigma^{\prime}$ indicates the hypothetical stress in the absence of damage [21, 30]. Rupture is identified from the softening branch of the nominal stress-strain curve as the last point before the complete loss of load-carrying capacity. When an element reaches the rupture conditions, the damage variable becomes $D_{D}=1$ and the element 430 is deleted from the FE model [19. 
The element post-necking plastic elongation $\bar{u}$ is given by the relationship

$$
\bar{u}(\bar{\varepsilon})=\frac{\bar{\varepsilon}-\bar{\varepsilon}_{\mathrm{N}}}{\bar{\varepsilon}_{\mathrm{F}}-\bar{\varepsilon}_{\mathrm{N}}} \bar{u}_{\mathrm{F}},
$$

with

$$
\bar{u}_{\mathrm{F}}=\lambda_{S} \lambda_{E} L_{E}\left(\bar{\varepsilon}_{\mathrm{F}}-\bar{\varepsilon}_{\mathrm{N}}\right),
$$

where $L_{E}$ is the characteristic element length. Pavlović et al. 21] introduced two parameters to remove the dependence of the plastic elongation at fracture $\bar{u}_{\mathrm{F}}$ on the element size: the element-type factor $\lambda_{E}$ and the mesh-refinement factor $\lambda_{S}$. The former is a constant that depends only on the element type, while the latter is a function of $L_{E}$ with respect to a reference value $L_{E, 0}$

$$
\lambda_{S}=\left\{\begin{array}{ll}
1 & \text { for } L_{E}=L_{E, 0} \\
f\left(L_{E}\right) & \text { for } L_{E} \neq L_{E, 0}
\end{array} .\right.
$$

The two parameters are calibrated performing simulations that reproduce the uniaxial tensile test, following an iterative procedure [30. In the first step, the dogbone specimen (geometry R0 from Fig. A.19) is discretised using square shell elements with $L_{E}=L_{E, 0}$ and $\lambda_{S}=1$, while $\lambda_{E}$ is obtained through an iterative approach. The initial value $\lambda_{E}=2.5$ [21] is used to evaluate the ductile damage evolution law $\left(D_{D}, \bar{u}\right)$, which is employed in the simulation of the tensile test, and the predicted nominal-stress strain curve is compared with the experimental data. The value for $\lambda_{E}$ is considered satisfactory if the percentage difference between experimental and numerical rupture strain, $\delta\left(\varepsilon_{\mathrm{R}}^{\text {nom }}\right)$, is lower than $3 \%$, otherwise $\lambda_{E}$ is modified by \pm 0.1 and the simulation is repeated. Once $\lambda_{E}$ is found, the simulation is repeated for a new element size $L_{E}=L_{E, i}$ and an assumed value $\lambda_{S}=\left(L_{E, 0} / L_{E, i}\right)^{1 / 3}$ 21]. In this second step, $\lambda_{E}$ is fixed and $\lambda_{S}$ is modified by \pm 0.01 until $\delta\left(\varepsilon_{\mathrm{R}}^{\text {nom }}\right)<3 \%$. The second step is reiterated for five values of $L_{E, i}$, and a second order polynomial is used to correlate $\lambda_{S}$ to $L_{E}$. The element sizes considered in this study are $L_{E, 0}=0.5 \mathrm{~mm}$ and $L_{E, i}=[1.25,1.00,0.2,0.1] \mathrm{mm}$. The calibrated parameters are $\lambda_{E}=2.8, \lambda_{S}=$ $0.1118 L_{E}^{2}-0.4768 L_{E}+1.2126$. 
The shear damage model is completed by a softening law relating the damage variable $D_{S}$ to the plastic elongation $\bar{u}$, based on experimental data from the shear test (curve S in Fig. 1). A linear evolutionary law is assumed to describe the rapid softening following the onset of necking

$$
\left\{\begin{array}{ll}
D_{S}=0, \bar{u}=0 & \text { at necking } \\
D_{S}=1, \bar{u}=\bar{u}_{\mathrm{F}, S} & \text { at failure }
\end{array} .\right.
$$

In the previous equation, $\bar{u}_{\mathrm{F}, S}$ depends on the element size and needs to be calibrated through simulations that reproduce the shear test, using the specimen geometry S (Fig. A.19). The initial value is calculated as

$$
\bar{u}_{\mathrm{F}, S}=L_{E}\left(\varepsilon_{\mathrm{F}, S}^{\mathrm{nom}}-\varepsilon_{\mathrm{N}, S}^{\mathrm{nom}}\right)
$$

where the values of strain at necking, $\varepsilon_{\mathrm{N}, S}^{\mathrm{nom}}$, and at failure, $\varepsilon_{\mathrm{F}, S}^{\mathrm{nom}}$, are read from the FE model. The actual value $\bar{u}_{\mathrm{F}, S}$ is found iteratively when the condition $\delta\left(\varepsilon_{\mathrm{F}, S}^{\mathrm{nom}}\right)<3 \%$ is satisfied. The process is repeated for different values of element size $L_{E}=[0.13,0.12,0.10,0.09,0.07] \mathrm{mm}$, and the fitting curve obtained is $\bar{u}_{\mathrm{F}, S}=-1.1564 L_{E}^{2}+0.1636 L_{E}+0.0055$. It is worth noting that the region interested by failure in the shear specimen $\mathrm{S}$ is much smaller than the necking area in the dogbone specimen $\mathrm{R} 0$, therefore a smaller range of element size was investigated.

\section{References}

[1] V. Aune, E. Fagerholt, K. O. Hauge, M. Langseth, T. Børvik, Experimental study on the response of thin aluminium and steel plates subjected to airblast loading, Int. J. Impact Eng. 90 (2016) 106-121. doi:10.1016/j.

[2] N. Mehreganian, L. A. Louca, G. S. Langdon, R. J. Curry, N. Abdul-Karim, The response of mild steel and armour steel plates to localised air-blast loading-comparison of numerical modelling techniques, Int. J. Impact Eng. 115 (2018) 81-93. doi:10.1016/j.ijimpeng.2018.01.010. 

of thin plates subjected to impulsive load: Part III - an update 25 years on, Int. J. Impact Eng. 107 (2017) 1339-1351. doi:10.1016/j.ijimpeng. 2016.06 .010

[4] R. G. Teeling-Smith, G. N. Nurick, The deformation and tearing of thin circular plates subjected to impulsive loads, Int. J. Impact Eng. 11 (1) (1991) 77-91. doi:10.1016/0734-743X (91) 90032-B.

[5] G. N. Nurick, G. C. Shave, The deformation and tearing of thin square plates subjected to impulsive loads - An experimental study, Int. J. Impact Eng. 18 (1) (1996) 99-116. doi:10.1016/0734-743X (95)00018-2

${ }_{475}$ [6] M. D. Olson, G. N. Nurick, J. R. Fagnan, Deformation and rupture of blast loaded square plates-predictions and experiments, Int. J. Impact Eng. 13 (2) (1993) 279-291. doi:10.1016/0734-743X (93) 90097-Q.

[7] D. Bonorchis, G. N. Nurick, The effect of welded boundaries on the response of rectangular hot-rolled mild steel plates subjected to localised blast loading, Int. J. Impact Eng. 34 (11) (2007) 1729-1738. doi:10. 1016/j.ijimpeng.2006.11.002.

[8] N. Jacob, G. N. Nurick, G. S. Langdon, The effect of stand-off distance on the failure of fully clamped circular mild steel plates subjected to blast loads, Eng. Struct. 29 (10) (2007) 2723-2736. doi:10.1016/j . engstruct. 2007.01 .021

[9] B. M. Thomas, The effect of boundary conditions on the failure of thin plates subjected to impulsive loading, Ph.D. thesis, University of Cape Town (1995).

[10] N. S. Rudrapatna, R. Vaziri, M. D. Olson, Deformation and failure of blast-loaded stiffened plates, Int. J. Impact Eng. 24 (5) (2000) 457-474. doi:10.1016/S0734-743X(99)00172-4. 
[11] G. S. Langdon, S. C. K. Yuen, G. N. Nurick, Experimental and numerical studies on the response of quadrangular stiffened plates. Part II: Localised blast loading, Int. J. Impact Eng. 31 (1) (2005) 85-111. doi:10.1016/j. ijimpeng.2003.09.050.

[12] N. Jones, Dynamic inelastic response of strain rate sensitive ductile plates due to large impact, dynamic pressure and explosive loadings, Int. J. Impact Eng. 74 (2014) 3-15. doi:10.1016/j.ijimpeng.2013.05.003.

[13] N. S. Rudrapatna, R. Vaziri, M. D. Olson, Deformation and failure of blastloaded square plates, Int. J. Impact Eng. 22 (4) (1999) 449-467. doi: 10.1016/S0734-743X (98) 00046-3.

[14] Y. Yuan, P. J. Tan, Deformation and failure of rectangular plates subjected to impulsive loadings, Int. J. Impact Eng. 59 (2013) 46-59. doi:10.1016/ j.ijimpeng.2013.03.009.

[15] V. Aune, G. Valsamos, F. Casadei, M. Larcher, M. Langseth, T. Børvik, Numerical study on the structural response of blast-loaded thin aluminium

(1) and steel plates, Int. J. Impact Eng. 99 (2017) 131-144. doi:10.1016/j. ijimpeng.2016.08.010.

[16] M. Imachi, S. Tanaka, T. Q. Bui, S. Oterkus, E. Oterkus, A computational approach based on ordinary state-based peridynamics with new transition bond for dynamic fracture analysis, Eng. Fract. Mech. 206 (2019) 359-374. doi:10.1016/j.engfracmech.2018.11.054

[17] T. H. Nguyen, T. Q. Bui, S. Hirose, Smoothing gradient damage model with evolving anisotropic nonlocal interactions tailored to low-order finite elements, Comput. Methods Appl. Mech. Eng. 328 (2018) 498-541. doi: $10.1016 / \mathrm{j} . \mathrm{cma} \cdot 2017.09 .019$

[18] C. T. Wu, T. Q. Bui, Y. Wu, T. L. Luo, M. Wang, C. C. Liao, P. Y. Chen, Y. S. Lai, Numerical and experimental validation of a particle Galerkin 
method for metal grinding simulation, Comput. Mech. 61 (3) (2018) 365383. doi:10.1007/s00466-017-1456-6.

[19] 3DS Dassault Systèmes, SIMULIA Abaqus 2017 Documentation, Dassault Systèmes, Providence, RI, 2016.

[20] Y. Bai, T. Wierzbicki, Application of extended Mohr-Coulomb criterion to ductile fracture, Int. J. Fract. 161 (1) (2010) 1-20. doi:10.1007/ s10704-009-9422-8.

[21] M. Pavlović, Z. Marković, M. Veljković, D. Bucrossed D Signevac, Bolted shear connectors vs. headed studs behaviour in push-out tests, J. Constr. Steel Res. 88 (2013) 134-149. doi:10.1016/j.jcsr.2013.05.003.

[22] Y. Li, M. Luo, J. Gerlach, T. Wierzbicki, Prediction of shear-induced fracture in sheet metal forming, J. Mater. Process. Technol. 210 (14) (2010) 1858-1869. doi:10.1016/j.jmatprotec.2010.06.021.

[23] M. Luo, T. Wierzbicki, Ductile fracture calibration and validation of anisotropic aluminum sheets, in: Soc. Exp. Mech. - SEM Annu. Conf. Expo. Exp. Appl. Mech. 2009, Vol. 1, 2009, pp. 302-312.

[24] M. Brünig, O. Chyra, D. Albrecht, L. Driemeier, M. Alves, A ductile damage criterion at various stress triaxialities, Int. J. Plast. 24 (10) (2008) 1731-1755. doi:10.1016/j.ijplas.2007.12.001

[25] L. Driemeier, M. Brünig, G. Micheli, M. Alves, Experiments on stresstriaxiality dependence of material behavior of aluminum alloys, Mech. Mater. 42 (2) (2010) 207-217. doi:10.1016/j.mechmat.2009.11.012.

[26] H. Hooputra, H. Gese, H. Dell, H. Werner, A comprehensive failure model for crashworthiness simulation of aluminium extrusions, Int. J. Crashworthiness 9 (5) (2004) 449-464. doi:10.1533/ijcr.2004.0289.

[27] N. Jones, A theoretical study of the dynamic plastic behavior of beams and plates with finite-deflections, Int. J. Solids Struct. 7 (8) (1971) 1007-1029. doi:10.1016/0020-7683(71)90078-3. 
[28] T. X. Yu, F. L. Chen, The large deflection dynamic plastic response of rectangular plates, Int. J. Impact Eng. 12 (4) (1992) 605-616. doi:10. 1016/0734-743X (92)90261-Q.

[29] A. C. Faralli, Large deformation of t-stub connection in bolted steel joints, Ph.D. thesis, UCL (University College London) (2019).

[30] A. C. Faralli, P. J. Tan, G. J. McShane, P. Wrobel, Deformation Maps for Bolted T-Stubs, J. Struct. Eng. (United States) 146 (5) (2020). doi: 10.1061/(ASCE) ST . 1943-541X.0002584. 